\title{
Advances in metabolic modeling of oleaginous microalgae
}

\author{
Juan D. Tibocha-Bonilla', Cristal Zuñiga ${ }^{2}$, Rubén D. Godoy-Silva ${ }^{1}$ and Karsten Zengler ${ }^{2,3,44^{*}}$
}

\begin{abstract}
Production of biofuels and bioenergy precursors by phototrophic microorganisms, such as microalgae and cyanobacteria, is a promising alternative to conventional fuels obtained from non-renewable resources. Several species of microalgae have been investigated as potential candidates for the production of biofuels, for the most part due to their exceptional metabolic capability to accumulate large quantities of lipids. Constraint-based modeling, a systems biology approach that accurately predicts the metabolic phenotype of phototrophs, has been deployed to identify suitable culture conditions as well as to explore genetic enhancement strategies for bioproduction. Core metabolic models were employed to gain insight into the central carbon metabolism in photosynthetic microorganisms. More recently, comprehensive genome-scale models, including organelle-specific information at high resolution, have been developed to gain new insight into the metabolism of phototrophic cell factories. Here, we review the current state of the art of constraint-based modeling and computational method development and discuss how advanced models led to increased prediction accuracy and thus improved lipid production in microalgae.
\end{abstract}

Keywords: Oleaginous phototrophs, Lipid production, Constraint-based metabolic modeling, Central carbon metabolism

\section{Background}

Photosynthetic microorganisms have been recognized as one of the oldest life forms on Earth [1]. These organisms, including microalgae such as Chlamydomonas sp., Synechocystis sp., and Chlorella sp., have attracted significant attention from the biotechnology industry because of their ability to efficiently transform renewable resources $\left(\mathrm{CO}_{2}\right.$, light, and water) into biomass and fuel precursors [2]. The photosynthetically produced biomass along with accumulated and secreted metabolites can be employed for the downstream synthesis of fuels (e.g., ethanol, biodiesel, and biocrude) and fine chemicals (e.g., pigments and organic acids) [3].

The world's ever-expanding requirement for cheap energy and fuel requires constant improvement of production platforms to meet the demand. The increased fuel consumption has led to an increase in global

\footnotetext{
*Correspondence: kzengler@ucsd.edu

2 Department of Pediatrics, University of California, San Diego, 9500

Gilman Drive, La Jolla, CA 92093-0760, USA

Full list of author information is available at the end of the article
}

greenhouse gas emissions [4], exemplified by a sharp increase in $\mathrm{CO}_{2}$ levels from $280 \mathrm{ppm}$ before the industrial revolution to today's $407 \mathrm{ppm}[5,6]$. Over $75 \%$ of these $\mathrm{CO}_{2}$ emissions have been attributed to the burning of fossil fuels $[7,8]$, rendering the reduction of humanity's carbon footprint a major global technological challenge. One alternative to address this challenge is increased utilization of biofuels from renewable resources and thus significant efforts have been underway to improve the efficiency of production of various biofuels [9].

Biofuels are categorized into first-, second-, and thirdgeneration biofuels depending on the type of raw material that is used for their production [10]. First-generation biofuels are produced from agricultural crops; one example being bioethanol production from sugarcane. These biofuels have been widely criticized as they pose extra demands on food production, which consequently raises food prices. Additionally, intensive agricultural processes to satisfy cost-effective production of crops for biofuels can lead to eutrophication and contamination of environmental resources $[8,11,12]$. As an alternative secondgeneration biofuels generated from woody waste and 
inedible food parts, such as biofuels from lignocellulosic biomass, have been proposed as a substitute for firstgeneration biofuels generated from food sources [10]. Secondary biofuels still require fertile land and often substantial amount of water for irrigation, limiting their areas of production. Third-generation biofuels, such as biosustainable production by microalgae, have thus been investigated to complement first- and second-generation biofuels. Third-generation biofuels also face several drawbacks which need to be overcome before turning into an economically viable alternative [13]. One of the largest challenges for third-generation biofuels from photosynthetic microorganisms lies in the harvesting process and downstream refinement of compounds of interest. For example, the costly recovery process of lipids from microalgal biomass, which in the case of biodiesel can account for up to $50 \%$ of the final cost [14], often prevents algae biofuel operations to be economically viable [14]. Higher lipid content would offset these staggering costs and would widely benefit the profitability and applicability of a third-generation biofuel technology. An early study by the US Department of Energy from 1978 reported that a lipid content of $60 \%$ would be necessary for third-generation biofuels to become economically feasible [15]. This number is now being revised to $20-40 \%$, depending on strain and cultivation conditions [16]. Increasing the lipid content of phototrophs has thus been a major focus for the biofuel industry. Major efforts to improve lipid content have been focused on optimizing culture conditions and on advanced strain engineering designs, both strategies of which greatly benefit from the use of metabolic modeling. In this review we compare various computational methods used for the rational design of strains and culture media, including flux balance analysis (FBA), dynamic flux balance analysis (dFBA), ${ }^{13} \mathrm{C}$ metabolic flux analysis $\left({ }^{13} \mathrm{C}\right.$ MFA), and elementary modes (EM) analysis. We focus in particular on the latest insights into central carbon metabolism (tricarboxylic acid cycle, the Calvin cycle, the glyoxylate shunt, glycolysis/gluconeogenesis, and the pentose-phosphate pathway) of oleaginous microalgae obtained by computational modeling as it is most relevant for production of biofuels and fuel precursors. Furthermore, we discuss the impact of time course modeling as well as the importance of incorporating compartmentalization into genome-scale models for microalgae and highlight the complexity of modeling lipid metabolism to increase biofuel productivity.

\section{Oleaginous photosynthetic microorganisms}

Microalgae have historically been classified into two classes: bacterial microalgae (Cyanophyta) and eukaryotic microalgae, the latter including green algae (Chlorophyta), red algae (Rhodophyta), and diatoms
(Bacillariophyta). Characteristic for all microalgae is their ability to grow photoautotrophically with $\mathrm{CO}_{2}$ and light as only carbon and energy sources. Several microalgae are also able to grow heterotrophically in the absence of light using various organic substrates, or grow mixotrophically, which refers to the uptake of organic carbon, e.g., glucose, sucrose, or acetate during growth in the light [17]. Oleaginous microalgae are attractive cell factories for the production of third-generation biofuels due to their ability to achieve an outstanding accumulation of lipids, in some cases surpassing $20 \%$ of total biomass in dry weight [13] and reaching economic feasibility [16]. Some studies have reported microalgae lipid productivities around 136,900 $\mathrm{L} \mathrm{ha}^{-1}$ year $^{-1}$ [12], which are several times higher than those achieved by oil palm plantations $\left(22,780 \mathrm{~L} \mathrm{ha}^{-1}\right.$ year $\left.^{-1}\right)[12,18]$. Microalgae have also been explored for the production of non-lipid-based biofuels [12]. Several genera of microalgae have been used for biofuel production, and metabolic models now exist for organisms such as Chlamydomonas [19-30], Chlorella [31-35], Nannochloropsis [36-38], Synechocystis [39-46], Tetraselmis [47], Monoraphidium [48], Ostreococcus [49], Tisochrysis [50], and Phaeodactylum [51-54]. The genetic tractability of several microalgae (Chlamydomonas, Synechocystis, Phaeodactylum) [55] also renders them interesting for gene-knockout studies using metabolic modeling tools. Metabolic models have enabled retrieving key information about central carbon metabolism, nutrient dependence, and distribution of reactions throughout different compartments in these organisms. Furthermore, dependence of carbon allocation on nutrient availability and the differential role of the main carbon pathways under several growth conditions have been revealed using these models. Examples for these findings will be discussed in detail below.

\section{Metabolic modeling}

Various modeling approaches have been deployed to improve the applicability of microorganisms for industrial applications. Modeling efforts can be categorized into isotope labeling-based, kinetic-based, and constraint-based approaches [56]. Isotope labeling studies and kinetic-based approaches are restricted to core metabolic networks or whole-cell analyses, although none of those methods is yet available on a genome scale and neither of these approaches considers organelle-specific compartmentalization. Constraint-based modeling approaches are currently the most widely used methods in metabolic modeling of oleaginous microalgae. These models enable in-depth understanding of microorganisms and their metabolism by simulating intracellular fluxes throughout a metabolic network, often at genome scale [57]. 
Genome-scale metabolic models (GSMs) are a mathematical representation of all the available biochemical and genomic information about a specific organism. GSMs have extensively been used to guide strain engineering designs by optimizing biochemical processes within an organism [33]. The reconstruction of a metabolic network can start de novo by identifying and adding reactions one by one, or it can be initiated by the creation of a draft reconstruction based on sequence homology to another related organism [33]. As of May 2018, 44 metabolic models for oleaginous microorganisms have been reported. Details about characteristics of available models are summarized in Table 1 . The highlights of milestones in metabolic modeling of oleaginous microalgae are shown in Fig. 1. While the first models for oleaginous microorganisms contained only core reaction, reaction size and complexity increased significantly over time (Fig. 1).

The first GSMs for oleaginous microalgae were reconstructed for Chlamydomonas reinhardtii [19] and Synechocystis sp. [41]. Reconstructing a GSM model requires high-quality information on genome sequence, gene function, and metabolism [58-60]. Manual curation is required to improve the accuracy of the model. This curation process is very time and labor intensive, often spanning weeks to months before completion. To facilitate rapid model generation, automated pipelines, such as ModelSEED [61] and PATRIC [62], have been made publicly available. ModelSEED and PATRIC are reconstruction tools based on subsystems annotation, in which metabolic networks are decomposed into subsystems and analyzed individually. Both tools are based on RAST (Rapid Annotations using Subsystems Technology) that compares the genome sequence with existing information from phylogenetic neighbors [63]. However, it has to be noted that reconstructions created by automated tools are prone to errors and special attention must be directed toward quality control and quality assurance (QC/QA) tests, in particular with regard to mass balance and energy production without input [57, 64]. Automatically and semi-automatically reconstructed models thus require intensive manual curation before detailed and accurate predictions can be made. Figure 2a compiles the number of core and genome-scale models created for oleaginous photosynthetic microorganisms reported to date.

All GSM models can be expressed as a general mass balance, which includes every metabolite being produced or consumed within the network in its respective reaction. This mass balance takes the form shown in Eq. (1):

$$
\frac{\mathrm{d}}{\mathrm{d} t} C=[S] \nu .
$$

The vector $C$ represents the instantaneous concentration of metabolites inside the cell, the vector contains all reaction rates and the matrix represents the stoichiometric information about reactions and participant metabolites. The stoichiometric matrix is a shared requirement among all constraint-based flux analysis approaches. Each column of this matrix contains the stoichiometric coefficients of a compound for all included reactions. In a similar fashion, each row represents the coefficients of all metabolites that take part in a single reaction [65]. An $m$ number of metabolites would render the $S$ matrix of $m \times n$ dimensions, with $n$ always greater than $m$.

The rectangular nature of the $\mathrm{S}$ matrix is one of the most important obstacles to overcome when working with metabolic networks and is easily seen when taking into account that for $m$ number of metabolites, there are $m$ change rates inside vector $C, m$ transport rates, and $p$ intracellular rates that are unknown. The system of equations then comprises only $m$ mass balances and as many as $n=2 m+p$ variables [66]. This system indetermination is what has given birth to several different approaches to metabolic modeling, which are discussed below. For system determination to be achieved, the measurement of a total of $m-n$ variables would be required. Large metabolic networks contain degrees of freedom that can amount to several hundreds. Therefore, the so-called core models, focusing on central metabolism, have been developed. These core models are used in metabolic flux analysis, such as the ${ }^{13} \mathrm{C}$-MFA, i.e., fluxomics. However, it is currently computationally infeasible to use large and compartmentalized metabolic networks for fluxomics analysis. Due to this, metabolic engineers have simplified the problem by transforming Eq. (1) into an optimization problem using an objective function and a defined set of constraints [65]. The definition of constraints results in a solution space, which delimits all possible functional states of a reconstructed network and a set of permitted phenotypes [67]. Metabolic models account for three types of constraints [65, 67]: (a) physico-chemical, which are based on conservation laws of mass and energy, dependency of reaction rates on biochemical loops and thermodynamics; (b) environmental, such as availability of nutrients, electron acceptors, and other external conditions (e.g. photon uptake); and (c) regulatory, including enzyme composition and performance, which helps to contextualize gene-related information, such as expression data and accurate gene-protein-reaction associations [68].

In phototrophic organisms, some physicochemical constraints are decided upon by following thermodynamic limits, regarding direction, reversibility or non-reversibility of reactions, which can be determined by calculating the Gibbs free energy. Environmental constraints are 
Table 1 Characteristics of current metabolic models of oleaginous microalgae

\begin{tabular}{|c|c|c|c|c|c|c|c|}
\hline Organism & Metabolic model (ID) & Analysis & Genes & Reactions & Metabolites & Compartments & $\begin{array}{l}\text { Citations } \\
\text { [references] }\end{array}$ \\
\hline Chlamydomonas reinhardtii & GSM & - & 1069 & - & - & - & $143[19]$ \\
\hline Chlamydomonas reinhardtii & GSM & - & - & 1500 & 1200 & - & $53[20]$ \\
\hline Chlamydomonas reinhardtii & GSM & FBA & - & 484 & 458 & 3 & $292[23]$ \\
\hline Chlamydomonas reinhardtii & GSM & FBA & - & 259 & - & 10 & $82[24]$ \\
\hline Chlamydomonas reinhardtii & GSM (AlgaGEM) & FBA & 2249 & 1725 & 1862 & 4 & $96[25]$ \\
\hline Chlamydomonas reinhardtii & GSM (iRC1080) & FBA & 1080 & 2190 & 1068 & 10 & $231[26]$ \\
\hline Chlamydomonas reinhardtii & $\mathrm{CM}$ & FBA & - & 280 & 278 & - & $47[27]$ \\
\hline Chlamydomonas reinhardtii & GSM & FBA & - & 160 & 164 & 2 & $100[28]$ \\
\hline Chlamydomonas reinhardtii & GSM & FBA & - & 280 & 278 & 0 & $12[29]^{a}$ \\
\hline Chlamydomonas reinhardtii & GSM (iBD1106) & FBA & 1106 & 2445 & 1959 & 10 & $10[30]^{b}$ \\
\hline Chlamydomonas reinhardtii & GSM (iCre1355) & FBA & 1355 & 2394 & 1133 & 10 & $12[21]$ \\
\hline Chlamydomonas reinhardtii & GSM & FBA ${ }^{13} C M F A$ & - & 139 & - & 3 & $2[22]$ \\
\hline Chlorella protothecoides & $\mathrm{CM}$ & ${ }^{13} \mathrm{CMFA}$ & - & 24 & 19 & 0 & $83[34]$ \\
\hline Chlorella protothecoides & GSM & FBA ${ }^{13} C M F A$ & 461 & 272 & - & 4 & $0[31]$ \\
\hline Chlorella pyrenoidosa & CM & MFA & - & 67 & - & 0 & $258[35]$ \\
\hline Chlorella sp. & CM & $\mathrm{dFBA}$ & - & 114 & 161 & - & $31[79]$ \\
\hline Chlorella variabilis & GSM (iAJ526) & FBA & 526 & 1455 & 1236 & 5 & $10[91]$ \\
\hline Chlorella vulgaris UTEX 395 & GSM (iCZ843) & FBA & 843 & 2294 & 1770 & 6 & $14[32]$ \\
\hline Chlorella vulgaris UTEX 396 & GSM (iCZ946) & $\mathrm{dFBA}$ & 946 & 2294 & 1770 & 6 & $2[33]^{c}$ \\
\hline Nannochloropsis gaditana & GSM (iRJ1321) & FBA & 1321 & 1918 & 1862 & 4 & $1[38]$ \\
\hline Nannochloropsis salina & GSM (iNS934) & $\mathrm{dFBA}$ & 934 & 2345 & - & 10 & $4[37]$ \\
\hline Nannochloropsis sp. & GSM & FBA & 383 & 987 & 1024 & 6 & $0[36]$ \\
\hline Ostreococcus lucimarinus & GSM & FBA & - & 964 & 1100 & 2 & 38 [49] \\
\hline Ostreococcus tauri & GSM & FBA & - & 871 & 1014 & 2 & $38[49]$ \\
\hline Phaeodactylum tricornutum & GSM & - & 151 & 88 & - & 5 & $289[51]$ \\
\hline Phaeodactylum tricornutum & GSM & FBA & - & - & - & 2 & $12[52]$ \\
\hline Phaeodactylum tricornutum & GSM & FBA & 607 & 849 & 587 & 6 & $27[53]$ \\
\hline Phaeodactylum tricornutum & GSM (iLB1027) & FBA & 1027 & 4456 & 2172 & 6 & $24[54]$ \\
\hline Synechococcus elongatus PCC7942 & GSM (iJB785) & FBA & 785 & 850 & 768 & 7 & $13[78]$ \\
\hline Synechococcus sp. PCC 7002 & GSM (iSyp611) & FBA & 611 & 552 & 542 & 2 & $39[92]$ \\
\hline Synechococcus sp. PCC 7002 & GSM (iSyp708) & FBA & 708 & 646 & 581 & 2 & $39[93]$ \\
\hline Synechococcus sp. PCC 7002 & GSM (iSyp821) & FBA & 821 & 792 & 777 & 3 & $3[94]$ \\
\hline Synechococcus sp. PCC 7002 & GSM (iSyp728) & FBA & 728 & 742 & 696 & 7 & 22 [95] \\
\hline Synechocystis sp. PCC 6803 & CM & ${ }^{13} \mathrm{CMFA}$ & - & 29 & - & - & $181[96]$ \\
\hline Synechocystis sp. PCC 6803 & CM & FBA & - & 70 & 46 & 2 & $165[39]$ \\
\hline Synechocystis sp. PCC 6803 & CM & FBA & - & 43 & - & - & $43[40]$ \\
\hline Synechocystis sp. PCC 6803 & GSM & FBA & - & 380 & 291 & 6 & $159[41]$ \\
\hline Synechocystis sp. PCC 6803 & GSM & FBA & 669 & 882 & 790 & 2 & $113[44]$ \\
\hline Synechocystis sp. PCC 6803 & GSM (iSyn811) & FBA & 811 & 956 & 911 & 2 & $59[43]$ \\
\hline Synechocystis sp. PCC 6803 & GSM & FBA ${ }^{13} C M F A$ & - & 493 & 465 & 2 & $51[42]$ \\
\hline Synechocystis sp. PCC 6803 & GSM (iJN678) & FBA & 678 & 863 & 795 & 3 & $206[46]$ \\
\hline Synechocystis sp. PCC 6803 & GSM & FBA & 677 & 759 & 601 & 6 & $143[45]$ \\
\hline Tetraselmis sp. & GSM & FBA & 2249 & 1725 & 1862 & 4 & $2[47]^{d}$ \\
\hline Tisochrysis lutea & CM & EM & - & 157 & 162 & 2 & $2[50]$ \\
\hline
\end{tabular}

Metabolic models are classified into two different groups: Genome-scale metabolic models (GSM) and core models (CM). The analyses were classified in: flux balance analysis (FBA), dynamic FBA (dFBA), elementary modes (EM), metabolic flux analysis (MFA), MFA using ${ }^{13} \mathrm{C}$ tracer $\left({ }^{13} \mathrm{C}\right.$ MFA), and their combinations

a Modified the metabolic model of $C$. reinhardtii from Cogne et al. [27]

b Modified the metabolic model of $C$. reinhardtii from Chang et al. [26]

c Used the genome-scale model of $C$. vulgaris from Zuñiga et al. [32]

d Used the genome-scale model of C. reinhardtii from Dal'Molin et al. [25] with constraints for Tetraselmis sp. 


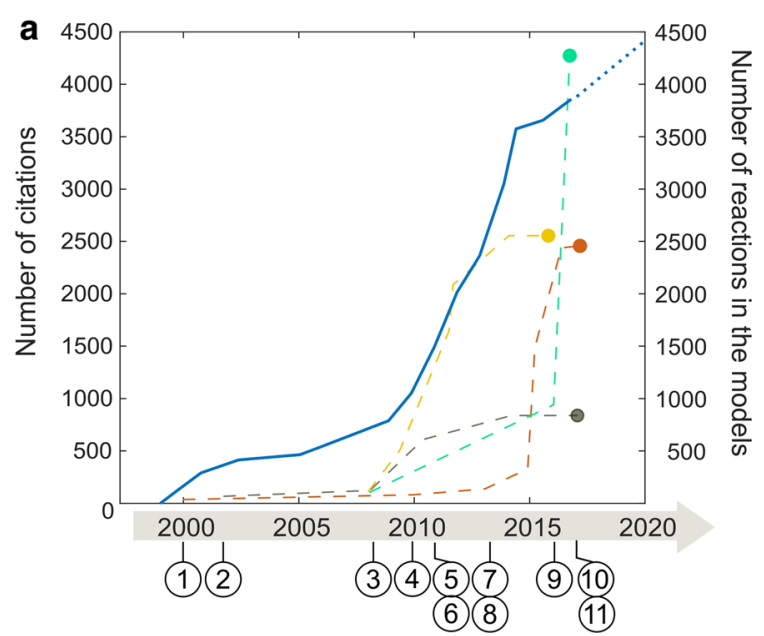

(1) 2000 First model for the oleaginous eukaryotic microalgae Chlorella pyrenoidosa [35]

(2) 2002 First model for the oleaginous prokaryotic microalgae Synechocystis sp. [96]

(3) 2008 First model for a diatom (Phaeodactylum tricornutum) [51]

(4) 2010 First GSM (Synechocystis sp.), containing more than 500 genes [41]

(5) 2011 Fluxomics study using ${ }^{13} \mathrm{C}$ MFA for Synechocystis sp. [42]

(6) 2011 Integration of light absorptions into the model for C. reinhardtii [24]

(7) 2013 Elemental composition analysis for Tisochrysis /utea [50]

(8) 2013 First application of dFBA to the core model for Chlorella sp. [80]

(9) 2016 Reconstruction of the most comprenhesive genome-scale model to date for a prokaryotic microalgae (S. elongtus PCC6803)* [43]

(10) 2017 Reconstruction of the most comprenhesive genome-scale models to date for eukaryotic microalgae (N. salina and C. vulgaris $)^{*}[32,37]$

(11) 2017 Introduction of dynamic biomass compositions for $C$. vulgaris, improving predictability of expression and proteomics data [33]

b
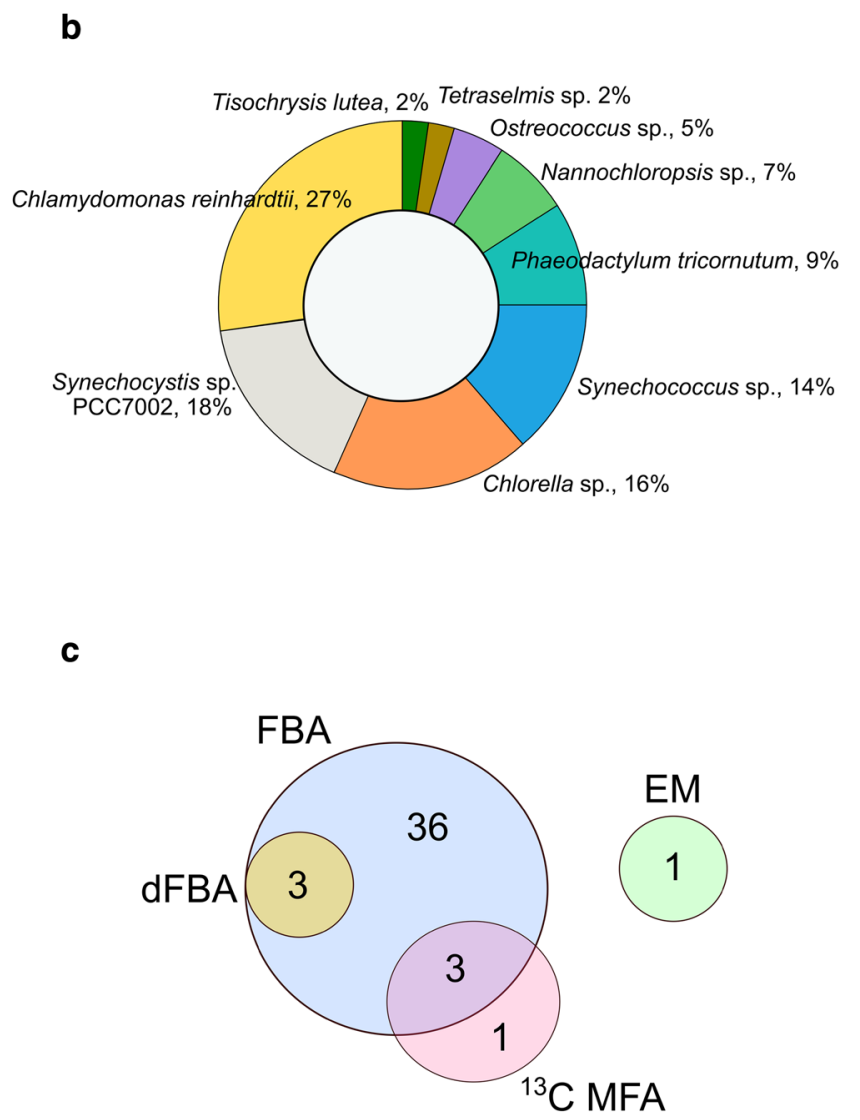

Fig. 1 Key developments in constraint-based metabolic modeling of oleaginous microalgae. a Cumulative number of citations for all 44 publications related to "Metabolic Modeling of Oleaginous Microalgae and Cyanobacteria" (blue line) and conservatively estimated future citations (blue dotted line). Dashed lines represent the number of reactions per model for Chlamydomonas (yellow), Synechocystis, and Synechococcus (gray), Chlorella (orange), Phaeodactylum (green). b Breakdown of the total number of publications by microorganism (percentage) highlights the importance of model organisms such as Synechocystis, Synechococcus, Chlorella, Chlamydomonas, and Chlorella. c Frequency of metabolic modeling approaches used to solve models for oleaginous microalgae: flux balance analysis (FBA), followed by ${ }^{13} \mathrm{C}$ metabolic flux analysis, dynamic flux balance analysis (dFBA), and elementary modes (EM)

usually based on measured experimental values of light quality, and nutrient and substrate uptake rates. Some regulatory constraints are those used in a study by Levering et al., in which the GSM of the diatom Phaeodactylum tricornutum was employed to capture the response to varying environmental conditions due to a transcriptional regulatory network [69]. Despite this, there are still too many variables to account for in the dynamic system. Various approaches to analyze the metabolic network of oleaginous microalgae are discussed below.

\section{Flux balance analysis (FBA)}

Most metabolic modeling studies involving oleaginous microalgae have been using FBA for simulation. A few other approaches have been used as an alternative or complement, such as ${ }^{13} \mathrm{C}$-MFA [22, 31, 34, 42] or EM [50]. Figure $1 \mathrm{~b}, \mathrm{c}$ highlights existing models and methods used to interrogate these models. Currently, large-scale metabolic networks are analyzed mainly in silico using FBA. Analysis of dynamic data obtained by experimentally intensive strategies like ${ }^{13} \mathrm{C}$-MFA rely on simplified metabolic models, e.g., representing only central metabolism [22, 31, 34, 42].

FBA refers to the application of linear programming to analyze fluxes under balanced metabolite conditions [65]. This statement is based on two assumptions: first, the cells are in steady state and, second, all cells have a general objective while growing. The first assumption simplifies the system significantly by neglecting all transient behavior of the metabolite concentrations, thus yielding Eq. (2). The elimination of all the unknown concentration change rates inside is mathematically convenient, but forces the system, i.e., a culture flask or bioreactor, to theoretically exist in a steady state. 


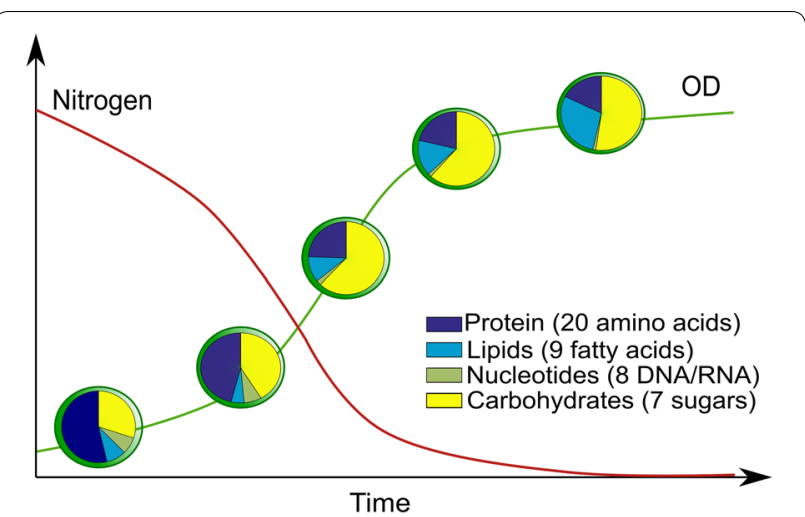

Fig. 2 Changing biomass composition (Chlorella vulgaris) in response to nitrogen depletion determined over time. While available nitrogen (red line) decreases and optical density (OD, green line) increases over a growth course, the microalga accumulates storage compounds. Accumulation of storage compounds, such as lipids and carbohydrates, leads to a reduction of total protein. Data collected from [32]

$$
[S] v=0
$$

The second assumption of an objective function in the model implies that all cells grow with a specific objective, which is the same for every cell during the calculation time. The most widely used objective function for FBA is the maximization of biomass production, which implies that the organism has evolved sufficiently to have the optimal arrangement of fluxes so that its growth will be maximized. While this assumption is likely correct for certain microorganisms, it is not universally applicable [70]. For example, under nutrient-deficient conditions the objective of a cell might not be biomass production, but rather the optimization of the production rate of storage compounds for later use. In a similar way, we know that phenotypic states vary in accordance with the growth phase or environmental conditions (Fig. 2), especially those that exhibit a dynamic biomass composition, such as phototrophs [71-73] and yeast [74]. Thus, timespecific biomass compositions are needed for light-dark cycles, considering degradation of storage pools during dark periods. This is of particular interest for the production of biofuel precursors. Furthermore, maximization of carbon uptake rate as $\mathrm{CO}_{2}$ has been proposed as a suitable objective function for autotrophic modeling during the light period [32]. FBA has proven to be useful and to reproduce overall experimental behavior in silico, although a true steady state is hardly encountered in experimental settings [58]. Its versatility and the accurate reproducibility of experimental results under several culture conditions make FBA one of the most widely used methods for metabolic modeling [75].

\section{Biomass objective function}

The biomass objective function (BOF) is a broadly used modeling reaction, which drives the supplemented resources across the metabolic network to produce all known cellular components in the model (such as amino acids, nucleotides, fatty acids, carbohydrates, vitamins, ions, and cofactors). Maximizing the BOF allows simulating growth rate and the yield of carbon source to biomass (henceforth referred to as biomass yield). The BOF can be determined from the genome sequence [59] or through experimentation. Both approaches have been successfully applied, especially for prokaryotic microorganisms. However, when microorganisms have been subjected to non-optimal conditions, such as extreme temperatures, $\mathrm{pH}$, or limited nutrient concentrations, a single BOF is often not suitable to predict experimental data successfully [70, 76]. For these cases, auxiliary objective functions have been proven necessary, such as minimization of ATP production, substrate uptake rate, or redox potential production rate [70].

There are several levels of refinement of the BOF [77], but it generally consists in the definition of a set of metabolites which compose the biomass. The set can be composed of just one reaction yielding a hypothetical compound called "biomass" or could otherwise be refined up to building blocks or biomass components (carbohydrates, lipids, proteins, DNA, RNA, pigments, etc.) [78]. The BOF of manually curated metabolic models of oleaginous microorganisms often accounts for hundreds of metabolites as part of the lipid metabolism, because of lipids being the primary target for biofuel production in these organisms. Lipid chain fatty acids (14:0, $16: 1,18: 1,16: 2)$ are usually summarized as triacylglycerols (TAG), monogalactosyldiacylglycerols (MGDG), etc., representing the entirety off all lipids in the organism. Accurate BOF composition has enabled the improved prediction of phenotypic states. It has been claimed that constrained BOF furthers the predictability of experimental nutrient- and light-limited conditions [33]. In some cases, the BOF has been complemented by a twostep optimization approach with minimization of uptake rates. In autotrophic growth conditions, minimization of light uptake (photons) has been employed but no significant improvement of the growth rate prediction has been obtained [23, 39]. In the same way, minimization of carbon source substrate uptake rate has been utilized for heterotrophic growth $[25,47]$. As alternatives, minimization of flux magnitudes across the network was used for P. tricornutum [51, 54], maximization of ATP yield [28], and minimization of ATP demand [24] for C. reinhardtii, and maximization of hydrogen production rate for both C. reinhardtii [25] and Synechocystis sp. [40]. 


\section{Dynamic FBA}

Overcoming the steady-state assumption of standard FBA is vital for the modeling of highly dynamic systems, which are characteristic of photosynthetic microorganisms [33, 37, 79]. These organisms have evolved under cyclic light/dark conditions, which require switching between different phenotypic states. During light periods, inorganic carbon is fixed into storage carbon compounds, such as carbohydrates and lipids, which are consumed in the dark period to accommodate vital cell functions. The storing-for-later behavior results in a dynamic biomass composition that can change during the light period (hours) or along the course of growth (days). In the case of C. vulgaris and other phototrophs, it has been shown that the biomass composition is also dependent on nitrogen availability (Fig. 2). Since FBA is used under a steady-state assumption, it is virtually disqualified for its use in the aforementioned cases. On the other hand, not including this assumption would add a set of ordinary differential equations to the problem, yielding a differential-algebraic system. To solve this, a dynamic FBA approach was proposed using either a dynamic optimization approach (DOA) or a static optimization approach (SOA) [80].

The DOA calculates the time profiles of fluxes and metabolite concentrations by solving the optimization problem over the entire time span of interest, running the calculation only once. The dynamic system is transformed into a non-linear programming problem (NLP) by parameterizing the differential equations through the method of orthogonal collocation on finite elements, described by Cuthrell and Biegler [81]. The BOF is then rewritten as a weighted average of the instantaneous and the terminal objective functions and is subjected to the system of differential equations along with the constraints. The SOA approach, on the other hand, solves the optimization problem multiple times, once for each time interval. At the end, an integration of the set of instantaneous rates of change over the interval is carried out for the calculation of metabolite concentrations.

Experiment-based BOF constraints are an alternative method to simulate dynamic metabolic behavior [33]. Changes in the BOF influence the state of the metabolic network, thus directly affecting predictions. This approach improved the accuracy of flux prediction by considering measurements over the course of growth under autotrophic and heterotrophic conditions in Chlorella vulgaris. The time series flux distributions accurately simulate $75 \%$ of expression and proteomics data collected over the course of growth, including allosteric reactions and multi-subunit enzymes. This approach also enabled the determination of the net content of nitrogen pools at each condition [33]. When an experimental determination of metabolites constituting the BOF is not feasible, unsteady-state methods, such as unsteady-state FBA (uFBA), can be applied. These unsteady-state methods operate with a limited number of measured metabolites. uFBA was recently developed and applied to study heterotrophic microorganisms [86], but uFBA would be a promising approach for the analysis of photosynthetic microorganisms.

\section{Unsteady-state FBA}

The aim of uFBA is to calculate internal flux distributions from existing time-course data, e.g., target metabolomics data. These datasets typically contain information about several (five to ten) metabolites such as glycerol, ethanol, and acetate. It is necessary to determine the rate of change of these metabolites from the experimental data and to include these rates in the system of equations [82]. Ideally, all rates of change would be known and the uFBA could be run as a series of standard FBA methods. Since this is often not feasible, all immeasurable variables are assumed to be, initially, under steady-state conditions as well as under a closed system assumption, i.e., with no possibility of transport inside or outside the cell. Elimination of this amount of transport reactions can often overdetermine the system and requires further conditioning. A "metabolite node relaxation" algorithm has been deployed that assigns sink reactions to unmeasured variables to allow for their accumulation or depletion. The algorithm is based on optimizations that find the minimum number of sink reactions that are necessary while keeping the model computable [86].

\section{Metabolic flux analysis (MFA)}

MFA is an alternative to FBA which also assumes a steady-state mass balance [83]. When working with small enough metabolic networks, it is possible to measure or define enough numbers of internal or external fluxes to determine the algebraic equation system. For this strategy, Eq. (2) is rewritten by decomposing the matrix and the vector into the measurable (known) and the immeasurable (unknown) fluxes, as shown in Eq. (3).

$$
[\boldsymbol{S}]_{u} v_{u}+[\boldsymbol{S}]_{m} v_{m}=0
$$

The larger the metabolic network, the more the fluxes are necessary to measure for system determination. Therefore, metabolic networks of several hundred reactions require measurements of internal fluxes for most of the fluxes, e.g. by ${ }^{13} \mathrm{C}$ labeling $[22,31,42]$.

\section{Elementary modes (EM)}

EM is based on the calculation of all the solutions of the system in Eq. (2) in the allowable flux space, restricting the solution with a thermodynamic constraint and a 
non-decomposability constraint [84]. The latter renders each solution an elementary flux mode, which means it is a unique and minimal set of reactions. These sets can be rewritten into macroscopic reactions, thus reducing the degrees of freedom exhibited formerly by Eq. (2). Often, EM is combined with core genome-scale metabolic models to provide energetic efficiencies and optimal flux distributions [84, 85]. The use of EM analysis (Fig. 1c) has declined over the last years, in part due to the rapid development of omic tools applied to sequencing, which enables generating genome-scale metabolic network reconstructions based on complete genome sequences.

\section{Lessons learned from metabolic modeling of oleaginous phototrophs}

Advances in modeling of microalgae are in part due to the availability of extensive omic datasets. Having full genome sequences available was crucial for generating the initial genome-scale metabolic models for the microalgae Chlamydomonas $[23,26]$ and opened the possibilities of creating additional algae models based on homology [32]. Fluxomic data has played a major role in elucidating central carbon metabolism of microalgae (e.g., C. protothecoides [34], C. reinhardtii [22], and Synechocystis sp. [42]). This tool has also served as a validation tool for GSM models [30]. In addition, metabolomics or transcriptomics have been used in context of the model to study and optimize biochemical pathways of industrial interest [86]. In this section, we describe different approaches to reconstruct and simulate metabolic models for oleaginous microalgae to increase growth and lipid content and improve bioproduction.

\section{Growth conditions}

Several microalgae are able to grow as autotrophs, heterotrophs, or mixotrophs. Some metabolic pathways are only active under certain growth modes, including the oxidative/reductive pentose phosphate pathway [22, 23, $27,39,40]$, Calvin cycle, and presumably the glyoxylate shunt [39]. Hence, differential mathematical models are necessary for correct prediction for each growth condition, requiring unique stoichiometric matrices and biomass formation equations. The study and prediction of phenotypes dependent on growth conditions is perhaps the most studied aspect regarding in oleaginous microalgae. Currently, the models accurately predict growth with hundreds of different nitrogen and carbon sources [30, 32]. Furthermore, most models are able to reproduce more than one growth mode, including mixotrophy in the models $i$ CZ843 [32], iCZ946 [33], iRJ1321 [38], $i$ RC1080 [26], AlgaGEM [25], iNS934 [37], iLB1027 [53], and a model for Nannochloropsis sp. [36].
Experimentally, highest biomass yields have been reported for autotrophic conditions, while lowest were obtained under heterotrophic growth in P. tricornutum [53], Synechocystis sp. [39], C. reinhardtii [23], and Chlorella sp. [79]. Mixotrophic growth, as a kind of hybrid condition, has shown biomass yields falling between ones observed for autotrophic and heterotrophic. However, an exception is the study of Navarro et al. [40], in which a mixotrophic biomass yield (92\%) higher than the autotrophic one $(60 \%)$ was predicted as reported for Synechocystis sp. The constraints regarding growth conditions directly affect the way carbon is distributed across the metabolic network, and thus the biomass yield and production rate. So, it is crucial to determine and adjust these constraints if needed for each growth condition. For example, under autotrophic growth the biomass yields have been reported to be close to $100 \%$, since no carbon is lost in the process [23, 39, 44, 53, 79], thus experimental measurements can be used directly. On the other hand, under heterotrophic growth conditions a significant carbon loss as $\mathrm{CO}_{2}$ in oleaginous microalgae has been reported to vary between 37\% [39] to 40\% for Synechocystis sp. [40], $50 \%$ for C. reinhardtii [23] and 50.5\% for Chlorella sp. [79] as a result of the carbon input flux being lost as $\mathrm{CO}_{2}$ due to energy production through the TCA cycle and the oxidative pentose phosphate pathway (PPP) [23, 39, 44, 53, 79]. Mixotrophic biomass yields tend to be higher than under heterotrophy, since part of the released $\mathrm{CO}_{2}$ is fixed once again [39]. Reported net biomass yields are therefore around 92\% (Synechocystis sp.), $100 \%$ (C. reinhardtii) and 80\% (Chlorella sp.) assuming a closed system.

\section{Light conditions}

Since light directly impacts microalgae growth and behavior, efforts have been made to define the quality and quantity of light constraints in metabolic models [29, 37]. Models can be significantly improved by considering a more realistic light uptake mechanism, since correctly defined constraints regarding light-driven reactions allow for the assessment of light influence on carbon allocation. Chang et al. [26] proposed dividing the total light spectrum into effective spectral bandwidths, each of which had an associated effective bandwidth coefficient. These coefficients, along with the activity spectra of light-driven reactions, allowed for the correct calculation of flux distribution along these reactions, taking into account that phototrophic organisms are strongly affected by the nature of the incoming light. Manually curated models account for reactions to simulate light sources, such as solar, incandescent, fluorescent, metal halide, highpressure sodium, red and white LED. High-resolution light phenomena in the model enables to determine the 
most suitable irradiance conditions for increased growth and lipid productivities. However, it has been shown that the bandwidth coefficient varies from microorganism to microorganism, as well as with culture size and growth vessels used, for example flasks, pilot, or industrial-scale vessels. Therefore, microorganism-specific refining for light uptake modeling in the GSM models will be needed for further improvement [39].

\section{Intracellular pools}

Although metabolic modeling has focused on simulating the intracellular environment of a cell under steady state, allowing the accumulation of certain metabolites in pools has proven necessary for the correct prediction of phenotypic states $[60,87]$. Metabolite pools can play an important role in the regulation of reactions, since different pathways find themselves interconnected by common metabolite collections. Target metabolomics data have been used successfully to constrain the metabolic model of Chlorella and determine the pool size of nitrogen [33]. The determination of the pool size was achieved by scanning the nitrogen uptake rate while fixing experimentally determined biomass compositions over the course of growth. Thereafter, nitrogen pool concentrations were computed by integrating the predicted nitrogen uptake rates necessary to meet the required biomass composition each time. Other target examples are energydependent and energy-replenishing processes which are coordinated by the ATP, ADP and AMP pools [87] as well as nitrogen and chrysolaminarin pools in P. tricornutum [54].

\section{Compartmentalization}

Eukaryotic microalgae contain different organelles (e.g., cytosol, mitochondria, nucleus, endoplasmic reticulum, glyoxysome, chloroplast, Golgi apparatus, vacuole, thylakoid, eye spot, and the cell wall). The exact compartmentalization is species dependent. Accurate annotation of proteins and compartmentalization in the model is necessary for maximizing information content and gaining detailed knowledge about microalgae metabolism. Flux distributions highly depend on the model's capability for metabolic exchange prediction between organelles. Careful manual curation of these models and delimitation of capabilities while adding reactions and reconstructing eukaryotic models in an automatic matter is thus crucial to achieve maximal predictability [63].

The example of nicotinamide adenine dinucleotide phosphate (NADPH) production in eukaryotic microalgae highlights the importance of compartmentalization. The PPP plays the role of producing NADPH in the cytosol, while the electron transport chain (ETC) is in charge of producing it in the chloroplast.
Non-compartmentalized models can predict that the entire NADPH demand is supplied by the ETC, rather than PPP supplying NADPH demand outside the chloroplast. This issue was encountered in the first metabolic model of an oleaginous microalgae C. pyrenoidosa [35]. While the model can simulate central carbon metabolism in general, it cannot predict detailed engineering targets since information about where fluxes take place is not available.

Early metabolic models were focused on the reconstruction of core algae models, which were later expanded to include genome-scale information (Table 1) $[19,20,35,49,50]$. The least compartmentalized model included only the chloroplast and cytosol, to uncouple the NADPH consumption/production of the Calvin cycle and the PPP [28]. More refined models now account for the mitochondria, thylakoid lumen, glyoxysome (peroxisome), extracellular environment, nucleus, Golgi apparatus, endoplasmic reticulum, vacuoles, and the cell wall $[21,24,26,30,36,37]$.

\section{Modeling lipid production}

Phototrophs produce several different kinds of lipids, including tri- and diglycerides, phospho- and glycolipids and hydrocarbons [14]. More specifically, lipid production in oleaginous microalgae includes triacylglycerol (TAG), phosphatidylglycerol, phosphatidylinositol, phosphatidylethanolamine, sulfoquinovosyldiglycerol, MGDG, digalactosyldiglycerol, and phosphatidylcholine. TAG alone can accumulate from 20 to $60 \%$ of dry cell weight in some species like $C$. vulgaris [32]. Modeling and gaining insight into the increased lipid content of microalgae has been the object of several studies. Most of these studies have investigated the effect of nitrogen depletion [21,33, 34, 37, 38, 42, 48, 54], while others have studied the influence of low $\mathrm{CO}_{2}$ and low light [54] on increasing overall lipid content. A study of Nannochloropsis gaditana reported increased lipid productivity under mixotrophic growth conditions [38].

When microalgae are subjected to nitrogen depletion conditions, carbon flux is drawn away from photosynthetic pathways as cells shift into the stationary phase and begin to store carbon as starch and lipids. This phenomenon and its effect on biomass composition are displayed in Fig. 2, in which a general trend of biomass composition evolution as a function of time and nitrogen availability is presented. Under these non-optimal culture conditions, microalgae shift the central carbon flux from biomass production to the production of storage compounds. As a result, the growth rate is decreased because carbohydrates and/or lipid are accumulated under stress conditions [40]. C. protothecoides was reported to redirect $81 \%$ of the input carbon flux toward fatty acid synthesis, but 
as a consequence decreased its growth rate by $30 \%$ [34]. In a similar way, $C$. reinhardtii was found to accumulate TAG when faced with nutrient limitation, but its growth halted after $8 \mathrm{~h}$ of cultivation [22]. Interestingly, Lim et al. [47] reported downregulation of TAG biosynthesis for Tetraselmis sp. after $24 \mathrm{~h}$, though TAG accumulation continued after this time point. The authors claimed this to be a result of decreased lipid degradation rather than lipid production.

\section{New insights into the central carbon metabolism of microalgae}

Most studies on oleaginous microalgae have focused on the central carbon metabolism and revealed new findings about glycolysis, PPP, TCA cycle, and the Calvin cycle. Figure 3 shows the most important metabolic pathways in microalgae and how they are linked to lipid metabolism. FBA has been used to study genome-wide fluxes through the metabolic network under mixotrophy, heterotrophy, and autotrophy. While most studies coincide in their assessment of functionality and fluxes in central carbon pathways, other pathways such as the glyoxylate and ornithine shunt are still not well understood, and modeling results are often not consistent between different studies and organisms [22, 23, 25, 31, $34,39,40,42,79]$. As a general rule, significant carbon flow through the TCA cycle has been reported under heterotrophic conditions, which demand catabolism of external organic compounds, contributing to the reduction of flux through the electron transport chain (ETC) and the Calvin cycle [23, 32, 35, 38]. During heterotrophic growth, most microalgae prefer glucose as carbon and energy source (Fig. 3). Other microalgae, such as $C$. reinhardtii, are only capable of assimilating two-carbon organic compounds, like acetate [22]. When glucose enters the cytosol, its fate can either be oxidation via glycolysis to pyruvate, oxidation via PPP to ribose 5-phosphate or transformation into storage compounds (lipids, glycogen and starch) [88]. In microalgae, acetate coming from the extracellular environment can be converted in the glyoxysome to succinate through the glyoxylate shunt, which can be considered as a variation of the TCA cycle. Succinate, an important biosynthetic precursor that can be converted into oxaloacetate, from which phosphoenolpyruvate (PEP) can be synthesized by the enzyme PEP carboxykinase, and enter gluconeogenesis for carbohydrate or lipid synthesis [17]. Under autotrophic growth, the carbon source is inorganic $\left(\mathrm{CO}_{2}\right)$ and the energy source is light. In the thylakoid lumen of eukaryotic microalgae, the ETC takes advantage of protons from light to store its energy in the form of ATP and NADPH, which are subsequently used to reduce $\mathrm{CO}_{2}$ molecules into triose phosphates (G3P) in the Calvin cycle. G3P can then

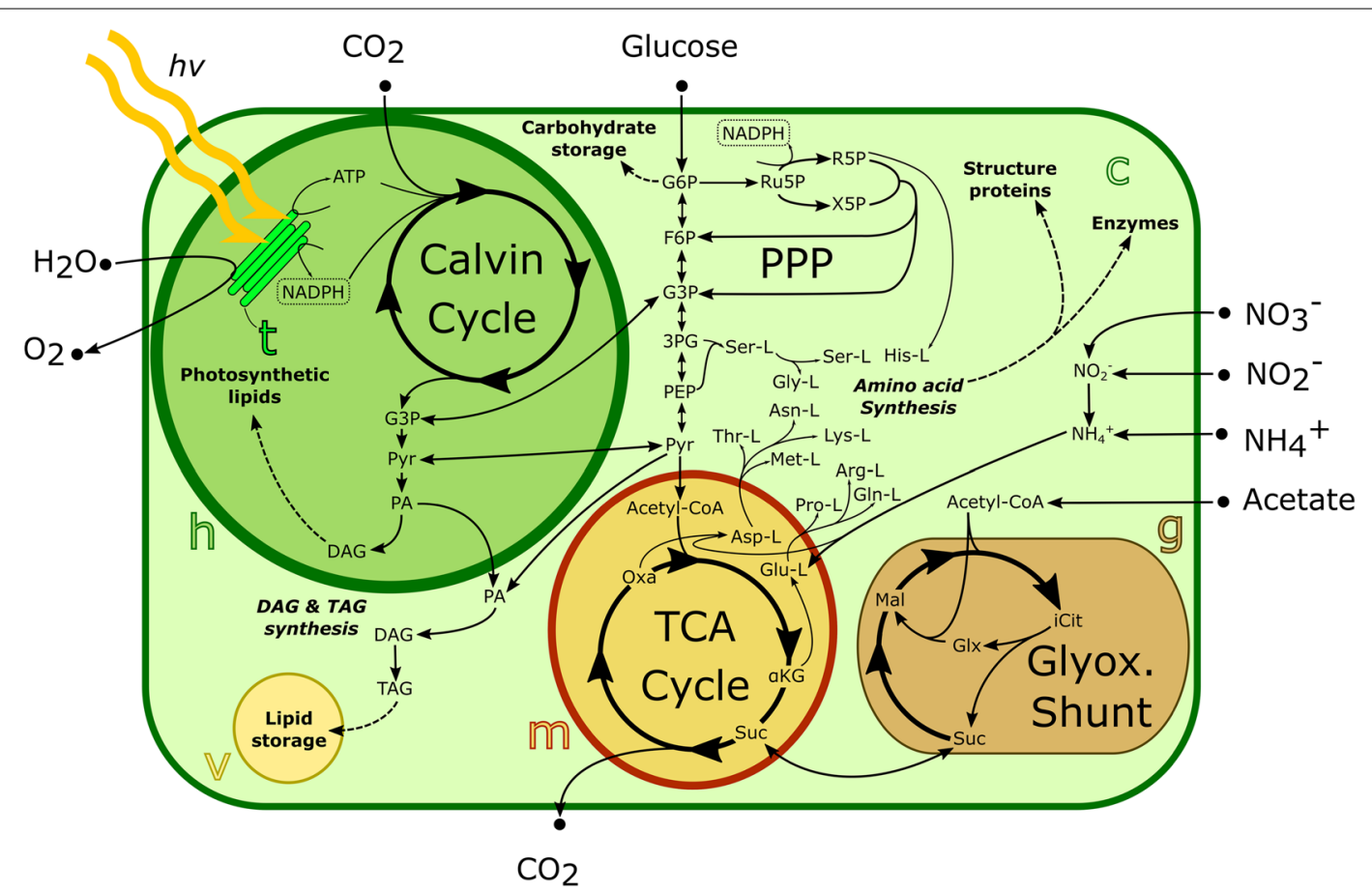

Fig. 3 Central metabolism in eukaryotic microalgae. The main compartments of active metabolism are shown, i.e., the chloroplast (h), thylakoid lumen (t), vacuole (v), mitochondrium (m), glyoxysome (g), and cytosol (c) 
be converted into glucose 6-phosphate (G6P), sucrose, starch and other sugars in the chloroplast.

\section{Tricarboxylic acid cycle}

The TCA accounts for the highest carbon fluxes and number of active reactions under heterotrophic growth conditions [32, 35]. Under this mode, the percentage of the total carbon input flux into the TCA cycle was reported to be $35.6 \%$ in $C$. reinhardtii grown with acetate [22] and $18.7 \%$ in C. protothecoides grown with glucose. However, under autotrophic and mixotrophic conditions, only half of the activity has been reported [79], with only
8-10 out of 22 reactions carrying flux for both microorganisms [32]. The role of the TCA under these conditions shifts to the production of biosynthetic precursors rather than energy production. Figure 4 shows complete and possible bypass variations of the TCA cycle observed in different photosynthetic microorganisms. Cogne et al. [27] reported that under autotrophic growth the TCA cycle in C. reinhardtii was operating as two branches with production of 2-oxoglutarate on one end, and malate on the other, with an input through oxaloacetate via the anaplerotic activity of the phosphoenolpyruvate carboxylase (Fig. 4). Zero flux was found through the

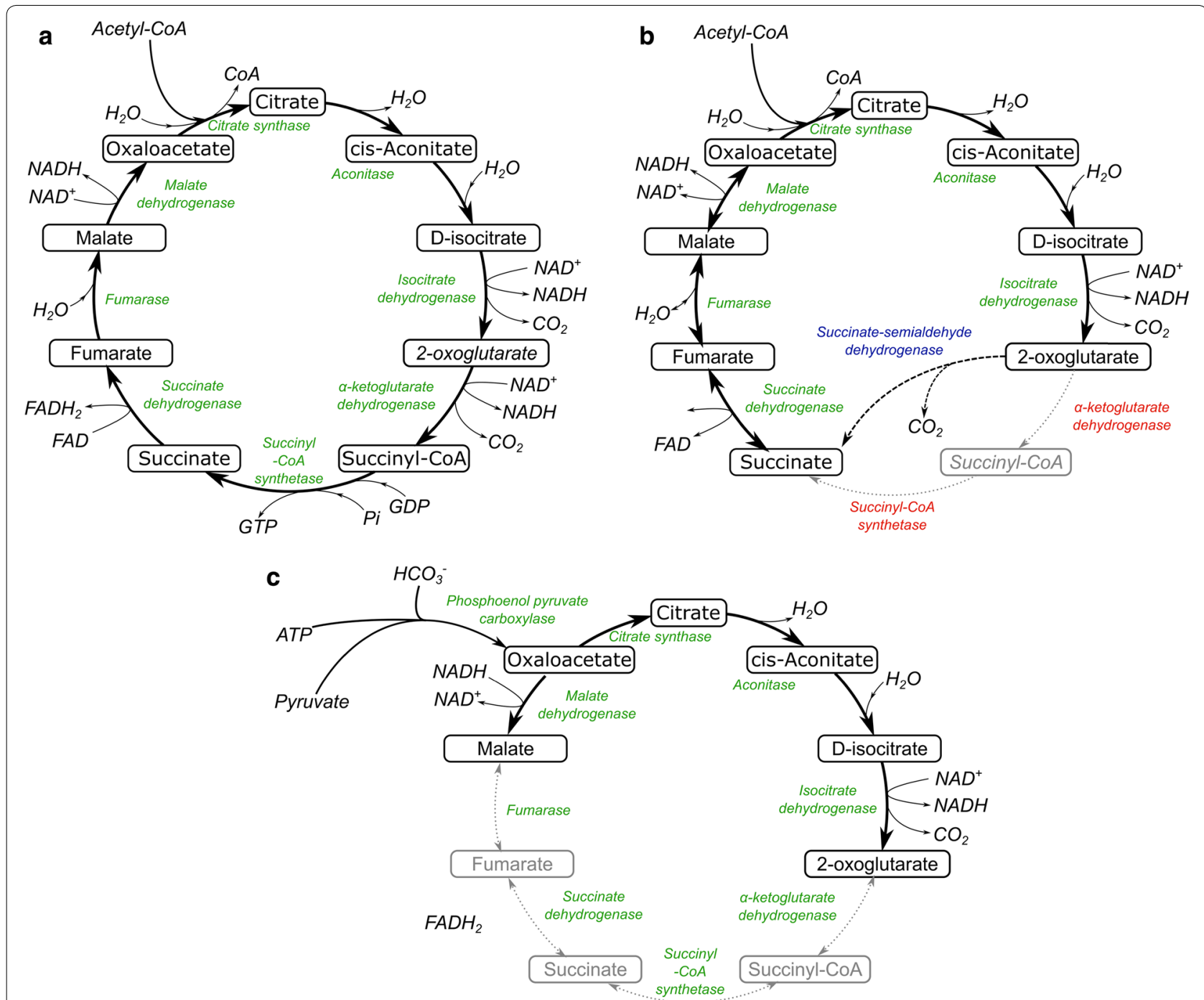

Fig. 4 Variations of the TCA cycle in photosynthetic microorganisms. a Complete and fully functional TCA cycle. b TCA cycle observed in microalgae, such as Synechococcus sp., which lacks the enzymes a-ketoglutarate dehydrogenase and succinyl-CoA synthetase (enzymes highlighted in red). A bypass via succinate-semialdehyde dehydrogenase, as observed in Synechocystis sp., is shown in blue. c Split TCA cycle as reported for C. reinhardtii [30]. The two branches producing 2-oxoglutarate and malate for downstream biosynthesis. Oxaloacetate is provided via anaplerotic activity of phosphoenolpyruvate carboxylase in this split TCA cycle [46] 
enzymes 2-oxoglutarate dehydrogenase, succinyl-CoA synthetase, and succinate dehydrogenase, since energy demands can be supplied by the PPP and the glyoxylate shunt. Other studies have also reported such similarities between prokaryotic and eukaryotic organisms [89, 90], in which prokaryotic microalgae, such as Synechocystis sp. and Synechococcus elongatus, do not possess a complete TCA cycle. These bacteria lack the $\alpha$-ketoglutarate (2-oxoglutarate) dehydrogenase and succinyl CoA synthetase $[17,78]$. Knoop et al. [41] have claimed a bypass via the succinate-semialdehyde dehydrogenase to compensate for the lack of 2-oxoglutarate dehydrogenase as shown in Fig. 4. The bypass replenishes intermediaries in the TCA cycle linked to lipids biosynthesis such as acetyl-CoA.

\section{Reductive/oxidative pentose phosphate pathway}

The oxidative and reductive phases of the PPP serve different purposes. While the oxidative phase serves as a catabolic pathway for NADPH production from the oxidation of G6P, the reductive phase represents an anabolic pathway of biosynthesis of 5-carbon carbohydrates for synthesis of nucleic acid, coenzyme A, ATP, and other biomass and lipids biosynthetic precursors [79]. High flux through the oxidative PPP means that energy production is being used for maintenance rather than growth [35]. It has been reported that depending on the growth conditions, either one phase can appear active. However, for the diatom $P$. tricornutum a low flux through the whole PPP pathway was determined. The reduced flux is explained with this organisms' ability to obtain 5-carbon carbohydrates via phosphopentose epimerase [53].

As a general result for non-compartmentalized models, energy-yielding oxidative PPP appears inactive during autotrophic growth, since the model predicts energy comes from the ETC in the form of NADPH rather than the dissimilatory pathways $[35,39]$. As stated above, NADPH demand outside the chloroplast should be supplied by the PPP rather than the ETC. However, the compartmentalized models of Cogne et al. [27] and Boyle and Morgan [23] predicted inactivation of the oxidative PPP for C. reinhardtii under autotrophic conditions. In the latter study, cells were found to prefer indirect energy transport by taking G3P from the chloroplast to the mitochondria and degrading it to 3-phosphoglycerate (3PG), releasing both ATP and NADH [23]. Furthermore, the fact that $C$. reinhardtii uses acetate as a carbon source instead of glucose greatly affects its phenotypic behavior and flux distribution under heterotrophy. Since the input to the PPP is G6P, incoming acetate would have to be transformed through several reactions in the glyoxylate shunt to oxaloacetate and then to G6P (Fig. 3). For this reason, NADPH production in $C$. reinhardtii preferably takes place via the ETC under autotrophic growth, while it is produced mainly through the glyoxylate shunt under heterotrophic growth [22, 23, 31, 34, 35, 39-41, 79]. Limitation in the transport or consumption of G6P or 3PG can result in metabolite accumulation, leading to the synthesis of certain types of lipids. For example, C. reinhardtii produces mainly triglyceride lipids.

Apart from growth conditions, other external factors have been reported to alter the flux distribution through the PPP. Wu et al. [31] found that increased oxygen availability in C. protothecoides decreases the flux through the PPP and instead enhances flux through the TCA cycle, thus producing more energy and yielding more $\mathrm{CO}_{2}$. Moreover, increased synthesis of storage compounds under nitrogen-depletion conditions was shown to increase PPP fluxes due to increased demand of NADPH for biosynthesis [34].

\section{Glyoxylate shunt}

The ability of the glyoxylate shunt of transforming acetyl-CoA into succinate for biosynthetic purposes renders it vital for the metabolism of acetate independent of its source, i.e. extracellular environment. However, the glyoxylate shunt has been found to be inactive under heterotrophic [31, 34, 79], autotrophic [39, 40, 79], or mixotrophic growth conditions [42] for various organisms, e.g., Synechococcus sp. In C. reinhardtii and $P$. tricornutum; however, the glyoxylate shunt has been reported to be active for all tested heterotrophic conditions [22, 23, 25]. The inactive glyoxylate shunt under autotrophic growth can be explained by the cell not taking up acetate from the environment, but rather synthesizing storage compounds, such as lipids and carbohydrates, that represent desirable bioproducts $[40,80]$.

\section{Calvin cycle}

Reducing equivalents and ATP formed in the ETC under autotrophic conditions are used later in the Calvin cycle to produce triose phosphates (G3P) for further synthesis of carbohydrates, which can be assimilated or turned into backbone structures of lipids. During autotrophic growth conditions, the entire anabolic activity relies on the Calvin cycle. G3P is transformed into higher carbohydrate molecules, such as pentoses and hexoses, through the PPP and gluconeogenesis, respectively. Moreover, lipid and amino acid anabolism is dependent on pyruvate produced from the G3P [88]. It has been reported in green algae that the Calvin cycle fixes $\mathrm{CO}_{2}$ in the form of 3PG, which gets converted to dihydroxyacetone phosphate (DHAP) subsequently [79]. Naturally, the Calvin cycle is inactive in the dark. When microalgae are subjected to mixotrophic conditions, carbohydrate demand poses a competition between uptake of external organic 
carbon sources and the Calvin cycle (i.e., inorganic carbon uptake). In C. reinhardtii the majority of carbon flux was found to be directed toward the Calvin cycle, rather than glycolysis and TCA under mixotrophic growth [23]. The cyanobacterium Synechocystis sp. however, was found to be dominated completely by the organic carbon uptake before a specific threshold of light intensity was surpassed. After this verge of irradiance, rubisco-dependent carboxylation and oxygenation were increased immediately and all Calvin cycle reactions were activated [41].

\section{Conclusions}

Great advances have been made in constraint-based modeling of photosynthetic microorganisms over the last two decades. Metabolic modeling has been proven critical for our understanding of complex metabolism in microalgae. Model-driven approaches have helped to identify boundaries for light and nutrient conditions as well as suitable genetic targets to increase lipid productivity. Metabolic models have progressed from core models to genome-scale metabolic models, which now include detailed compartmentalization and light uptake. Furthermore, the dynamic behavior and rapidly changing phenotypes due to changing environmental parameters are important traits of these organisms and have now been included in model simulations. Those recent extensions and improvements allow elucidating phenotypic behavior under different growth and culture conditions over time. In addition, these new models provide a high-quality standard for the improvements of existing metabolic models as well as for future reconstructions. Despite extensive efforts on refinement and manual curation of metabolic models, there are still open questions regarding the central metabolism and dynamic biomass composition in microalgae. Coupling metabolic modeling with fluxomic experiments can improve our knowledge of the activity of the glyoxylate shunt and ornithine shunt. Furthermore, time course-dependent expression datasets are needed to constrain and validate the models and to gain insight into the dynamics of metabolism. These datasets will enable to broaden the scope of the models and to elucidate missing transport reactions. Multi-omics dataset can also increase the predictability of carbon exchange and storage within the cell and guide improved production of desirable compounds in microalgae.

\footnotetext{
Abbreviations

ADP: adenosine diphosphate; AMP: adenosine monophosphate; ATP: adenosine triphosphate; BOF: biomass objective function; C: metabolite concentration vector; $\mathrm{CBFA}$ : constraint-based flux analysis; $\mathrm{CO}_{2}$ : carbon dioxide; $\mathrm{CoA}$ : coenzyme A; dFBA: dynamic flux balance analysis; DHAP: dihydroxyacetone phosphate; DNA: deoxyribonucleic acid; EM: elementary modes; ETC: electron transport chain; FBA: flux balance analysis; G3P: glyceraldehyde-3-phosphate;
}

G6P: glucose-6-phosphate; GSM: genome-scale metabolic (model); MGDG: monogalactosyldiacylglycerols; MFA: metabolic flux analysis; NADH: nicotinamide adenine dinucleotide; NADPH: nicotinamide adenine dinucleotide phosphate; NLP: non-linear programming; PEP: phosphoenolpyruvate; PPP: pentose phosphate pathway; QA: quality assurance; QC: quality control; RNA: ribonucleic acid; S: stoichiometric matrix; TAG: triacylglycerols; TCA: tricarboxylic acid (cycle); uFBA: unsteady flux balance analysis.

\section{Authors' contributions \\ JT analyzed data; JT and CZ generated figures and tables; JT, CZ, RG, and KZ} wrote the manuscript. All authors read and approved the final manuscript.

\section{Author details}

${ }^{1}$ Grupo de Investigación en Procesos Químicos y Bioquímicos, Departamento de Ingeniería Química y Ambiental, Universidad Nacional de Colombia, Av. Carrera 30 No. 45-03, Bogotá, D.C., Colombia. ${ }^{2}$ Department of Pediatrics, University of California, San Diego, 9500 Gilman Drive, La Jolla, CA 92093-0760, USA. ${ }^{3}$ Department of Bioengineering, University of California, San Diego, 9500 Gilman Drive, La Jolla, CA 92093-0412, USA. ${ }^{4}$ Center for Microbiome Innovation, University of California, San Diego, 9500 Gilman Drive, La Jolla, CA 92093-0436, USA.

\section{Acknowledgements \\ JT would like to thank Ana Isabel Ramos Murillo for fruitful discussions.}

Competing interests

The authors declare that they have no competing interests.

\section{Availability of data and materials}

Not applicable.

\section{Consent for publication}

Not applicable.

\section{Ethics approval and consent to participate}

Not applicable.

\section{Funding}

This material is based on work supported by the National Science Foundation under Grants No. 1332344 and 1804187, the U.S. Department of Energy (DOE), Office of Science, Office of Biological and Environmental Research under Awards DE-SC0012658 and DE-SC0018344, and the Gordon and Betty Moore Foundation under grant GBMF7000.

\section{Publisher's Note}

Springer Nature remains neutral with regard to jurisdictional claims in published maps and institutional affiliations.

Received: 5 July 2018 Accepted: 27 August 2018

Published online: 05 September 2018

\section{References}

1. Falkowski PG, Raven JA. An introduction to photosynthesis in aquatic systems. In: Elworthy S, editor. Aquatic photosynthesis. Princeton: Princeton University Press; 2013. p. 1-43.

2. Gavrilescu M, Chisti Y. Biotechnology - a sustainable alternative for chemical industry. Biotechnol Adv. 2005;23:471-99.

3. Ghasemi Y, Rasoul-Amini S, Fotooh-Abadi E. The biotransformation, biodegradation, and bioremediation of organic compounds by microalgae. J Phycol. 2011;47:969-80.

4. EPA. Atmospheric concentrations of greenhouse gases. Environmental panel agency (EPA). https://www.epa.gov/climate-indicators/clima te-change-indicators-atmospheric-concentrations-greenhouse-gases. Accessed 2 Apr 2018.

5. Etheridge DM, Steele LP, Langenfelds RJRL, Francey RJ, Barnola JM, Morgan VI. Historical $\mathrm{CO}_{2}$ records from the Law Dome DE08, DE08-2, and DSS ice cores. In trends: a compendium of data on global change. http://cdiac .ess-dive.lbl.gov/trends/co2/lawdome.html. Accessed 6 Dec 2017. 
6. NASA. Climate change: vital signs of the planet: carbon dioxide. 2017. https://climate.nasa.gov/vital-signs/carbon-dioxide/. Accessed 6 Dec 2017

7. Marland G, Boden TA, Andres RJ, Brenkert AL. Global, regional, and national fossil fuel $\mathrm{CO}_{2}$ emissions. A compendium of data on global change. http://cdiac.ess-dive.lbl.gov/trends/emis/overview.html. Accessed 6 Dec 2017.

8. Brennan L, Owende P. Biofuels from microalgae — a review of technologies for production, processing, and extractions of biofuels and coproducts. Renew Sustain Energy Rev. 2010;14:557-77.

9. EPA. The sources and solutions: fossil fuels. 2015. https://www.epa.gov/ nutrientpollution/sources-and-solutions-fossil-fuels. Accessed 15 Nov 2017.

10. European Academies Science Advisory Council. The current status of biofuels in the European Union, their environmental impacts and future prospects. http://www.easac.eu/fileadmin/PDF_s/reports_statements/ Easac_12_Biofuels_Complete.pdf. Accessed 6 Dec 2017.

11. Rulli MC, Bellomi D, Cazzoli A, De Carolis G, D'Odorico P. The water-landfood nexus of first-generation biofuels. Sci Rep. 2016;6:22521.

12. Chisti Y. Biodiesel from microalgae. Trends Biotechnol. 2008;26:126-31.

13. Sandeep K. Sub- and supercritical water technology for biofuels. In: Lee JW, editor. Advanced biofuels and bioproducts. New York: Springer; 2013. p. 147-83.

14. Greenwell HC, Laurens LML, Shields RJ, Lovitt RW, Flynn KJ. Placing microalgae on the biofuels priority list: a review of the technological challenges. J R Soc Interface. 2009;7:703-26.

15. Sheehan J, Dunahay T, Benemann J, Roessler P. Look back at the U.S. Department of energy's aquatic species program: biodiesel from algae. https://www.nrel.gov/docs/legosti/fy98/24190.pdf. Accessed 30 Nov 2017

16. Muylaert K, Bastiaens L, Vandamme D, Gouveia L. Harvesting of microalgae: overview of process options and their strengths and drawbacks. Microalgae-based biofuels and bioproducts. Lisbon: Woodhead Publishing; 2017. p. 113-32.

17. Lee RE. Basic characteristics of the algae. In: Lee RE, editor. Phycology. New York: Cambridge University Press; 2008. p. 3-30.

18. Serrano-Bermúdez LM, Serrano Bermúdez LM. Estudio de cuatro cepas nativas de microalgas para evaluar su potencial uso en la producción de biodiesel. 2012. http://www.bdigital.unal.edu.co/7825/1/299883.2012. pdf. Accessed 10 June 2017.

19. May P, Wienkoop S, Kempa S, Usadel B, Christian N, Rupprecht J, et al. Metabolomics- and proteomics-assisted genome annotation and analysis of the draft metabolic network of Chlamydomonas reinhardtii. Genetics. 2008;179:157-66.

20. Christian N, May P, Kempa S, HandorfT, Ebenhöh O. An integrative approach towards completing genome-scale metabolic networks. Mol BioSyst. 2009;5:1889.

21. Imam S, Schäuble S, Valenzuela J, De Lomana ALG, Carter W, Price $N D$, et al. A refined genome-scale reconstruction of Chlamydomonas metabolism provides a platform for systems-level analyses. Plant J. 2015:84:1239-56.

22. Boyle NR, Sengupta N, Morgan JA. Metabolic flux analysis of heterotrophic growth in Chlamydomonas reinhardtii. PLOS ONE. 2017;12:e0177292.

23. Boyle NR, Morgan JA. Flux balance analysis of primary metabolism in Chlamydomonas reinhardtii. BMC Syst Biol. 2009;3:4.

24. Manichaikul A, Ghamsari L, Hom EFY, Lin C, Murray RR, Chang RL, et al. Metabolic network analysis integrated with transcript verification for sequenced genomes. Natl Inst Health. 2009;6:589-92.

25. De Dal'Molin CG, Quek L-E, Palfreyman RW, Nielsen LK. AlgaGEM-a genome-scale metabolic reconstruction of algae based on the Chlamydomonas reinhardtii genome. BMC Genomics. 2011;12(Suppl 4):5.

26. Chang RL, Ghamsari L, Manichaikul A, Hom EFY, Balaji S, Fu W, et al. Metabolic network reconstruction of Chlamydomonas offers insight into light-driven algal metabolism. Mol Syst Biol. 2011;7:518.

27. Cogne G, Rügen M, Bockmayr A, Titica M, Dussap CG, Cornet JF, et al. A model-based method for investigating bioenergetic processes in autotrophically growing eukaryotic microalgae: application to the green algae Chlamydomonas reinhardtii. Biotechnol Prog. 2011;27:631-40

28. Kliphuis AMJ, Klok AJ, Martens DE, Lamers PP, Janssen M, Wijfels RH. Metabolic modeling of Chlamydomonas reinhardtii: energy requirements for photoautotrophic growth and maintenance. J Appl Phycol. 2012;24:253-66.

29. Rügen M, Bockmayr A, Legrand J, Cogne G. Network reduction in metabolic pathway analysis: elucidation of the key pathways involved in the photoautotrophic growth of the green alga Chlamydomonas reinhardtii. Metab Eng. 2012;14:458-67.

30. Chaiboonchoe A, Dohai BS, Cai H, Nelson DR, Jijakli K, Salehi-Ashtiani K. Microalgal metabolic network model refinement through high-throughput functional metabolic profiling. Front Bioeng Biotechnol. 2014;2:1-12.

31. Wu C, Xiong W, Dai J, Wu Q. Genome-based metabolic mapping and 13C flux analysis reveal systematic properties of anoleaginous microalga Chlorella protothecoides. Plant Physiol. 2015;167:586-99.

32. Zuñiga C, Li C-T, Huelsman T, Levering J, Zielinski DC, McConnell BO, et al. Genome-scale metabolic model for the green alga Chlorella vulgaris UTEX 395 accurately predicts phenotypes under autotrophic, heterotrophic, and mixotrophic growth Conditions. Plant Physiol. 2016;172:589-602.

33. Zuñiga C, Levering J, Antoniewicz MR, Guarnieri MT, Betenbaugh MJ, Zengler $\mathrm{K}$, et al. Predicting dynamic metabolic demands in the photosynthetic eukaryote Chlorella vulgaris. Plant Physiol. 2017;176:450-62.

34. Xiong W, Liu L, Wu C, Yang C, Wu Q. ${ }^{13} \mathrm{C}$-tracer and gas chromatography-mass spectrometry analyses reveal metabolic flux distribution in the oleaginous microalga Chlorella protothecoides. Plant Physiol. 2010;154:1001-11.

35. Yang C, Hua Q, Shimizu K. Energetics and carbon metabolism during growth of microalgal cells under photoautotrophic, mixotrophic and cyclic light-autotrophic/dark-heterotrophic conditions. Biochem Eng J. 2000;6:87-102

36. Pham N. Genome-scale constraint-based metabolic modeling and analysis of Nannochloropsis sp. 2016. https://brage.bibsys.no/xmlui/bitst ream/handle/11250/2399905/12511_FULLTEXT.pdf?sequence=1 \&isAll owed $=\mathrm{y}$. Accessed 4 Dec 2017.

37. Loira N, Mendoza S, Paz Cortés M, Rojas N, Travisany D, Di Genova A, et al. Reconstruction of the microalga Nannochloropsis salina genome-scale metabolic model with applications to lipid production. BMC Syst Biol. 2017;11:66.

38. Shah AR, Ahmad A, Srivastava S, Jaffar Ali BM. Reconstruction and analysis of a genome-scale metabolic model of Nannochloropsis gaditana. Algal Res. 2017:26:354-64.

39. Shastri A, Morgan J. Flux balance analysis of photoautotrophic metabolism. Biotechnol Prog. 2005;21:1617-26.

40. Navarro E, Montagud A, Fernández de Córdoba P, Urchueguía JF. Metabolic flux analysis of the hydrogen production potential in Synechocystis sp. PCC6803. Int J Hydrogen Energy. 2009;34:8828-38.

41. Knoop H, Zilliges Y, Lockau W, Steuer R. The metabolic network of Synechocystis sp. PCC 6803: systemic properties of autotrophic growth. Plant Physiol. 2010;154:410-22.

42. Yoshikawa K, Kojima Y, Nakajima T, Furusawa C, Hirasawa T, Shimizu H. Reconstruction and verification of a genome-scale metabolic model for Synechocystis sp. PCC6803. Appl Microbiol Biotechnol. 2011;92:347-58.

43. Montagud A, Zelezniak A, Navarro E, de Córdoba PF, Urchueguía JF, Pati KR. Flux coupling and transcriptional regulation within the metabolic network of the photosynthetic bacterium Synechocystis sp. PCC6803. Biotechnol J. 2011:6:330-42

44. Montagud A, Navarro E, Fernández de Córdoba P, Urchueguía JF, Patil K. Reconstruction and analysis of genome-scale metabolic model of a photosynthetic bacterium. BMC Syst Biol. 2010;4:156.

45. Knoop H, Gründel M, Zilliges Y, Lehmann R, Hoffmann S, Lockau W, et al. Flux balance analysis of cyanobacterial metabolism: the metabolic network of Synechocystis sp. PCC 6803. PLoS Comput Biol. 2013;9:e1003081.

46. Nogales J, Gudmundsson S, Knight EM, Palsson BO, Thiele I. Detailing the optimality of photosynthesis in cyanobacteria through systems biology analysis. Proc Natl Acad Sci. 2012;109:2678-83.

47. Lim DKY, Schuhmann H, Thomas-Hall SR, Chan KCK, Wass TJ, Aguilera F, et al. RNA-Seq and metabolic flux analysis of Tetraselmis sp. M8 during nitrogen starvation reveals a two-stage lipid accumulation mechanism. Bioresour Technol. 2017:244:1281-93.

48. Bogen C, Al-Dilaimi A, Albersmeier A, Wichmann J, Grundmann M, Rupp $\mathrm{O}$, et al. Reconstruction of the lipid metabolism for the microalga Monoraphidium neglectum from its genome sequence reveals characteristics suitable for biofuel production. BMC Genomics. 2013;14:926. 
49. Krumholz EW, Yang H, Weisenhorn P, Henry CS, Libourel IGL. Genomewide metabolic network reconstruction of the picoalga Ostreococcus. J Exp Bot. 2012;63:2353-62.

50. Baroukh C, Muñoz-Tamayo R, Steyer JP, Bernard O. A new framework for metabolic modeling under non-balanced growth. Application to carbon metabolism of unicellular microalgae. PLOS ONE. 2013;12:107-12.

51. Kroth PG, Chiovitti A, Gruber A, Martin-Jezequel V, MockT, Parker MS, et al. A model for carbohydrate metabolism in the diatom Phaeodactylum tricornutum deduced from comparative whole genome analysis. PLoS ONE. 2008;3:e1426.

52. Singh D, Carlson R, Fell D, Poolman M. Modelling metabolism of the diatom Phaeodactylum tricornutum. Biochem Soc Trans. 2015;43:1182-6.

53. Kim J, Fabris M, Baart G, Kim MK, Goossens A, Vyverman W, et al. Flux balance analysis of primary metabolism in the diatom Phaeodactylum tricornutum. Plant J. 2016;85:161-76.

54. Levering J, Broddrick J, Dupont CL, Peers G, Beeri K, Mayers J, et al. Genome-scale model reveals metabolic basis of biomass partitioning in a model diatom. PLoS ONE. 2016;11:1-22.

55. Banerjee A, Banerjee C, Negi S, Chang J-S, Shukla P. Improvements in algal lipid production: a systems biology and gene editing approach. Crit Rev Biotechnol. 2017;38:369-85.

56. Cuperlović-Culf M. Dynamic metabolic profiling and metabolite network and pathways modeling. In: Nakamura K, editor. NMR metabolomics in cancer research. Oxford: Woodhead Publishing; 2013. p. 365-83.

57. Tan J, Zuñiga C, Zengler K. Unraveling interactions in microbial communities-from co-cultures to microbiomes. J Microbiol. 2015;53:295-305.

58. Orth JD, Thiele I, Palsson B $\varnothing$. What is flux balance analysis? Nat Biotechnol. 2010;28:245-8.

59. Thiele I, Palsson $B \varnothing$. A protocol for generating a high-quality genomescale metabolic reconstruction. Nat Protoc. 2010;5:93-121.

60. Feist $A M$, Palsson $B \varnothing$. The growing scope of application of genomescale metabolic reconstructions: the case of E. coli. Nat Biotechnol. 2008;26:659-67.

61. Overbeek R, Begley T, Butler RM, Choudhuri JV, Chuang HY, Cohoon M, et al. The subsystems approach to genome annotation and its use in the project to annotate 1000 genomes. Nucleic Acids Res. 2005;33:5691-702.

62. Snyder EE, Kampanya N, Lu J, Nordberg EK, Karur HR, Shukla M, et al. PATRIC: the VBI PathoSystems resource integration center. Nucleic Acids Res. 2007;35:D401-6.

63. Aziz RK, Bartels D, Best AA, DeJongh M, Disz T, Edwards RA, et al. The RAST server: rapid annotations using subsystems technology. BMC Genomics. 2008;9:75.

64. Fritzemeier CJ, Hartleb D, Szappanos B, Papp B, Lercher MJ. Erroneous energy-generating cycles in published genome scale metabolic networks: identification and removal. PLoS Comput Biol. 2017;13:1-14.

65. Terzer M, Maynard ND, Covert MW, Stelling J. Genome-scale metabolic networks. Wiley Interdiscip Rev Biol Med. 2009;1:285-97.

66. Villadsen J, Nielsen J, Lidén G. Biochemical reaction networks. In: Nielsen J, Villadsen J, editors. Bioreaction engineering principles. 3rd ed. Berlin: Springer; 2011. p. 151-214.

67. Price ND, Papin JA, Schilling CH, Palsson BO. Genome-scale microbial in silico models: the constraints-based approach. Trends Biotechnol. 2003:21:162-9

68. Chen K, Gao Y, Mih N, O'Brien EJ, Yang L, Palsson BO. Thermosensitivity of growth is determined by chaperone-mediated proteome reallocation. Proc Natl Acad Sci. 2017;114:11548-53.

69. Levering J, Dupont CL, Allen AE, Palsson BO, Zengler K. Integrated regulatory and metabolic networks of the marine diatom Phaeodactylum tricornutum predict the response to rising $\mathrm{CO}_{2}$ levels. mSystems. 2017:2:e00142-216

70. Takahashi O, Park Y-I, Nakamura Y. Biotechnology of microalgae, based on molecular biology and biochemistry of eukaryotic algae and cyanobacteria. FEBS Lett. 2009;583:3882-90.

71. Wan MX, Wang RM, Xia JL, Rosenberg JN, Nie ZY, Kobayashi N, et al. Physiological evaluation of a new Chlorella sorokiniana isolate for its biomass production and lipid accumulation in photoautotrophic and heterotrophic cultures. Biotechnol Bioeng. 2012;109:1958-64.

72. Toledo-Cervantes A, Garduño Solórzano G, Campos JE, MartínezGarcía M, Morales M. Characterization of Scenedesmus obtusiusculus AT-UAM for high-energy molecules accumulation: deeper insight into biotechnological potential of strains of the same species. Biotechnol Rep. 2018;17:16-23.

73. Cabello J, Morales M, Revah S. Dynamic photosynthetic response of the microalga Scenedesmus obtusiusculus to light intensity perturbations. Chem Eng J. 2014;252:104-11.

74. Dikicioglu D, Kırdar B, Oliver SG. Biomass composition: the "elephant in the room" of metabolic modelling. Metabolomics. 2015;11:1690-701.

75. García Sánchez CE, Torres Sáez RG. Comparison and analysis of objective functions in flux balance analysis. Biotechnol Prog. 2014;30:985-91.

76. Serrano-Bermúdez LM, González Barrios AF, Maranas CD, Montoya D. Clostridium butyricum maximizes growth while minimizing enzyme usage and ATP production: metabolic flux distribution of a strain cultured in glycerol. BMC Syst Biol. 2017;11:1-13.

77. Feist AM, Palsson BO. The biomass objective function. Curr Opin Microbiol. 2010;13:344-9.

78. Broddrick JT, Rubin BE, Welkie DG, Du N, Mih N, Diamond S, et al. Unique attributes of cyanobacterial metabolism revealed by improved genomescale metabolic modeling and essential gene analysis. Proc Natl Acad Sci. 2016;113:E8344-53

79. Muthuraj M, Palabhanvi B, Misra S, Kumar V, Sivalingavasu K, Das D. Flux balance analysis of Chlorella sp. FC2 IITG under photoautotrophic and heterotrophic growth conditions. Photosynth Res. 2013;118:167-79.

80. Mahadevan R, Edwards JS, Doyle FJ. Dynamic flux balance analysis of diauxic growth in Escherichia coli. Biophys J. 2002;83:1331-40.

81. Cuthrell JE, Biegler LT. On the optimization of differential-algebraic process systems. AlChE J. 1987;33:1257-70.

82. Bordbar A, Yurkovich JT, Paglia G, Rolfsson O, Sigurjónsson ÓE, Palsson BO. Elucidating dynamic metabolic physiology through network integration of quantitative time-course metabolomics. Sci Rep. 2017:7:1-12.

83. Wiechert W. 13C metabolic flux analysis. Metab Eng. 2001;3:195-206.

84. Trinh CT, Wlaschin A, Srienc F. Elementary mode analysis: a useful metabolic pathway analysis tool for characterizing cellular metabolism. Appl Microbiol Biotechnol. 2009;81:813-26.

85. Baroukh C, Muñoz-Tamayo R, Bernard O, Steyer JP. Mathematical modeling of unicellular microalgae and cyanobacteria metabolism for biofuel production. Curr Opin Biotechnol. 2015;33:198-205.

86. De Bhowmick G, Koduru L, Sen R. Metabolic pathway engineering towards enhancing microalgal lipid biosynthesis for biofuel applicationa review. Renew Sustain Energy Rev. 2015;50:1239-53.

87. Nikolaev EV, Burgard AP, Maranas CD. Elucidation and structural analysis of conserved pools for genome-scale metabolic reconstructions. Biophys J. 2005:88:37-49.

88. Nelson DL, Cox MM. Glycolysis, gluconeogenesis, and the pentose phosphate pathway. In: Freeman WH, editor. Lehninger principles of biochemistry. 4th ed. New York: Cox Publisher; 2008. p. 521-60.

89. Coronil T, Lara C, Guerrero MG. Shift in carbon flow and stimulation of amino-acid turnover induced by nitrate and ammonium assimilation in Anacystis nidulans. Planta. 1993;189:461-7.

90. Rai AK. Symbiotic systems with cyanobacteria-cyanobioses. In: Rai AK editor. Cyanobacterial nitrogen metabolism and environmental biotechnology. Chennai: Narosa Pub House; 1997. p. 299.

91. Juneja A, Chaplen FWR, Murthy GS. Genome scale metabolic reconstruction of Chlorella variabilis for exploring its metabolic potential for biofuels. Bioresour Technol. 2015;213:103-10.

92. Hamilton JJ, Reed JL. Identification of functional differences in metabolic networks using comparative genomics and constraint-based models. PLOS ONE. 2012;7:e34670.

93. Vu TT, Hill EA, Kucek LA, Konopka AE, Beliaev AS, Reed JL. Computational evaluation of Synechococcus sp. PCC 7002 metabolism for chemical production. Biotechnol J. 2013:8:619-30.

94. Qian X, Kim MK, Kumaraswamy GK, Agarwal A, Lun DS, Dismukes GC. Flux balance analysis of photoautotrophic metabolism: uncovering new biological details of subsystems involved in cyanobacterial photosynthesis. Biochim Biophys Acta Bioenerg. 2016;1858:276-87.

95. Hendry JI, Prasannan CB, Joshi A, Dasgupta S, Wangikar PP. Metabolic model of Synechococcus sp. PCC 7002: prediction of flux distribution and network modification for enhanced biofuel production. Bioresour Technol. 2016;213:190-7.

96. Yang C. Metabolic flux analysis in Synechocystis using isotope distribution from 13C-labeled glucose. Metab Eng. 2002:4:202-16. 